Imperial/TP/2009/JG/02

DESY 09-006

\title{
Supersymmetric solutions for non-relativistic holography
}

\author{
Aristomenis Donos ${ }^{1}$ and Jerome P. Gauntlett ${ }^{2}$ \\ ${ }^{1}$ DESY Theory Group, DESY Hamburg \\ Notkestrasse 85, D 22603 Hamburg, Germany \\ 2 Theoretical Physics Group, Blackett Laboratory, \\ Imperial College, London SW7 2AZ, U.K. \\ ${ }^{2}$ The Institute for Mathematical Sciences, \\ Imperial College, London SW7 2PE, U.K.
}

\begin{abstract}
We construct families of supersymmetric solutions of type IIB and $D=11$ supergravity that are invariant under the non-relativistic conformal algebra for various values of dynamical exponent $z \geq 4$ and $z \geq 3$, respectively. The solutions are based on five- and seven-dimensional Sasaki-Einstein manifolds and generalise the known solutions with dynamical exponent $z=4$ for the type IIB case and $z=3$ for the $D=11$ case, respectively.
\end{abstract}




\section{Introduction}

There has recently been much interest in finding holographic realisations of systems invariant under the non-relativistic conformal algebra starting with the work [1, [2] and discussed further in related work [3]-32. Such systems are invariant under Galilean transformations, generated by time and spatial translations, spatial rotations, Galilean boosts and a mass operator, which is a central element of the algebra, combined with scale transformations. If $x^{+}$is the time coordinate, and $\mathbf{x}$ denotes $d$ spatial coordinates, the scaling symmetry acts as

$$
\mathbf{x} \rightarrow \mu \mathbf{x}, \quad x^{+} \rightarrow \mu^{z} x^{+},
$$

where $z$ is called the dynamical exponent. When $z=2$ this non-relativistic conformal symmetry can be enlarged to an invariance under the Schrödinger algebra which includes an additional special conformal generator.

The solutions found in [1], [2] with $d=2$ and $z=2$ were subsequently embedded into type IIB string theory in [8, 9, [10] and were based on an arbitrary five-dimensional Sasaki-Einstein manifold, $S E_{5}$. The work of 9 also constructed type IIB solutions with $d=2$ and $z=4$ and again these were constructed using an arbitrary $S E_{5}$. It was also shown in [9] that the solutions with $z=2$ and $z=4$ can be obtained from a five dimensional theory with a massive vector field after a Kaluza-Klein reduction on the $S E_{5}$ space [9]. This procedure was generalised to solutions of $D=11$ supergravity in [31]: using a similar KK reduction on an arbitrary seven-dimensional Sasaki-Einstein space, $S E_{7}$, solutions with non relativistic conformal symmetry with $d=1$ and $z=3$ were found.

The type IIB solution of [8], [9], [10] with $z=2$ do not preserve any supersymmetry [9]. One aim of this note is to show that, by contrast, the type IIB solutions of [9] with $z=4$ and the $D=11$ solutions of [31] with $z=3$ are both supersymmetric and generically preserve two supersymmetries. A second aim is to generalise both of these supersymmetric solutions to different values of $z$. We will construct new supersymmetric solutions using eigenmodes of the Laplacian acting on one-forms on the $S E_{5}$ or $S E_{7}$ space. If the eiegenvalue is $\mu$ then we obtain type IIB solutions with $z=1+\sqrt{1+\mu}$ and $D=11$ solutions with $z=1+\frac{1}{2} \sqrt{4+\mu}$. This gives rise to type IIB solutions with $z \geq 4$ and $D=11$ solutions with $z \geq 3$, respectively. For the case

of $S^{5}$ we get solutions with $z=4,5, \ldots$ while for the case of $S^{7}$ we get solutions with $z=3,3 \frac{1}{2}, 4, \ldots$ and both of these preserve 8 supersymmetries.

Our constructions have some similarities with the construction of type IIB solutions in [24] that were based on eigenmodes of the Laplacian acting on scalar functions 
on the $S E_{5}$ space. Our IIB solutions preserve the same supersymmetry and we show how our solutions can be superposed with those of [24] while maintaining a scaling symmetry. An analogous superposition is possible for the $D=11$ solutions, which we shall also describe.

\section{The type IIB solutions}

The ansatz for the type IIB solutions we shall consider is given by

$$
\begin{aligned}
d s^{2} & =\frac{d r^{2}}{r^{2}}+r^{2}\left[2 d x^{+} d x^{-}+d x_{1}^{2}+d x_{2}^{2}\right]+d s^{2}\left(S E_{5}\right)+2 r^{2} C d x^{+} \\
F_{5} & =4 r^{3} d x^{+} \wedge d x^{-} \wedge d r \wedge d x_{1} \wedge d x_{2}+4 \operatorname{Vol}\left(S E_{5}\right) \\
& -d x^{+} \wedge\left[*_{C Y_{3}} d C+d\left(r^{4} C\right) \wedge d x_{1} \wedge d x_{2}\right]
\end{aligned}
$$

where $S E_{5}$ is an arbitrary five-dimensional Sasaki-Einstein space and the metric $d s^{2}\left(S E_{5}\right)$ is normalised so that the Ricci tensor is equal to four times the metric (i.e. the same normalisation as that of a unit radius five-sphere). Recall that the metric cone over the $S E_{5}$,

$$
d s^{2}\left(C Y_{3}\right)=d r^{2}+r^{2} d s^{2}\left(S E_{5}\right)
$$

is Calabi-Yau. The Kähler form on the $C Y_{3}$ is denoted $\omega_{i j}$ and the complex structure is defined 1 by $J_{i}{ }^{j}=\omega_{i k} g^{k j}$, where $g_{i j}$ is the Calabi-Yau cone metric. We will define the one-form $\eta$, which is dual to the Reeb vector on $S E_{5}$ by

$$
\eta_{i}=-J_{i}^{j}(d \log r)_{j}
$$

The one-form $C$ is a one-form on the $C Y_{3}$ cone. When $C=0$ we have the standard $A d S_{5} \times S E_{5}$ solution of type IIB which, in general, preserves eight supersymmetries (four Poincaré and four superconformal), corresponding to an $N=1 \mathrm{SCFT}$ in $d=4$. More generally, we can deform this solution by choosing $C \neq 0$ provided that $d C$ is co-closed on $\mathrm{CY}_{3}$ :

$$
d *_{C Y} d C=0
$$

With this condition, $F_{5}$ is closed and in fact it is also sufficient for the type IIB Einstein equations to be satisfied. As we will show these solutions preserve one

\footnotetext{
${ }^{1}$ While this is standard in the physics literature, often in the maths literature $J_{i}{ }^{j}=-\omega_{i k} g^{k j}$.
} 
half of the Poincaré supersymmetries. Note that the solution is invariant under the transformation

$$
x^{-} \rightarrow x^{-}-\Lambda, \quad C \rightarrow C+d \Lambda
$$

for some function $\Lambda$ on the CY cone. Thus, if $d C=0$, we can remove $C$, at least locally, by such a transformation.

We will look for solutions where the one-form $C$ has weight $\lambda$ under the action of $r \partial_{r}$. Then it is straightforward to check, following [1] and [2] that our solution is invariant under non-relativistic conformal transformations with two spatial dimensions $x^{1}, x^{2}$ and dynamical exponent $z=2+\lambda$. For example the scaling symmetry is acting as in (1.1) combined with $r \rightarrow \mu^{-1} r, x^{-} \rightarrow \mu^{2-z} x^{-}$. Following the analysis of closed and co-closed two forms on cones (such as $d C$ ) in appendix A of [33] we consider solutions constructed from a co-closed one-form $\beta$ on the $S E_{5}$ space that is an eigenmode of the Laplacian $\Delta_{S E}=\left(d^{\dagger} d+d d^{\dagger}\right)_{S E}$ :

$$
C=r^{\lambda} \beta, \quad \Delta_{S E} \beta=\mu \beta, \quad d^{\dagger} \beta=0 .
$$

It is straightforward to check that $d C$ is co-closed providing that $\mu=\lambda(\lambda+2)$. For our applications we choose the branch $\lambda=-1+\sqrt{1+\mu}$ leading to solutions with

$$
z=1+\sqrt{1+\mu}
$$

A general result valid for any five-dimensional Einstein space, normalised as we have, is that for co-closed 1 -forms $\mu \geq 8$ and $\mu=8$ holds iff the 1 -form is dual to a Killing vector (see section 4.3 of [34]). Thus in general our construction leads to solutions with

$$
z \geq 4
$$

Since all $S E_{5}$ manifolds have at least the Reeb Killing vector, dual to the one-form $\eta$, this bound is always saturated. Indeed the solution of [9] with $z=4$ is in our class. Specifically it can be obtained by setting $C=\sigma r^{2} \eta$ (and redefining $x^{-} \rightarrow-x^{-} / 2$ ): one can explicitly check that $\eta$ is co-closed on $S E_{5}$ and is an eigenmode of $\Delta_{S E}$ with eigenvalue $\mu=8$. Note that for this solution the two-form $d C$ is proportional to the Kähler-form of the Calabi-Yau cone: $d C=2 \sigma \omega$.

On $S^{5}$ the spectrum of $\Delta_{S^{5}}$ acting on one-forms is well known and we have $\mu=$ $(s+1)(s+3)$ for $s=1,2,3 \ldots$ (see for example [35] eq (2.20)) leading to $\lambda=s+1$ and hence new classes of solutions with $z=4,5,6 \ldots$ Note that these solutions come in families, transforming in the $S O(6)$ irreps $\mathbf{1 5}, \mathbf{6 4}, \mathbf{1 7 5}, \ldots$ To obtain similar results for $T^{1,1}$ one can consult [36]. 
We now discuss a construction that can be used when the spectrum of the Laplacian acting on functions is known, but not acting on one-forms. For example, the scalar Laplacian was studied in [40] for the $Y^{p, q}$ metrics [41], but as far as we know it has not been discussed acting on one-forms. Specifically we construct $(1,1)$ forms $d C$ on the $\mathrm{CY}$ cone using scalar functions $\Phi$ on the cone as follows. We write

$$
C_{i}=J_{i}{ }^{j} \partial_{j} \Phi
$$

for some function $\Phi$ on $C Y_{3}$. A short calculation shows that if

$$
\nabla_{C Y}^{2} \Phi=\alpha
$$

for some constant $\alpha$ then $d C$ is co-closed. The two-form $d C$ is a $(1,1)$ form on $C Y_{3}$ and it is primitive, $J^{i j} d C_{i j}=0$, if and only if $\alpha=0$. Observe that the solution of [9] with $z=4$ fits into this class by taking $\Phi=-\sigma r^{2} / 2$ and $\alpha=-6 \sigma$, leading to $C=\sigma r^{2} \eta$.

We now consider solutions with $\alpha=0$, corresponding to harmonic functions 2 on the CY cone with $d C(1,1)$ and primitive. We next write

$$
\Phi=r^{\lambda} f
$$

where $f$ is a function on the $S E_{5}$ space satisfying

$$
-\nabla_{S E_{5}}^{2} f=k f
$$

with $k=\lambda(\lambda+4)$ (see e.g. [37]). For the solutions of interest we choose the branch $\lambda=-2+\sqrt{4+k}$ leading to $z=\sqrt{4+k}$. For the special case of the five-sphere we can check with the results that we obtained above. The eigenfunctions $f$ on the five-sphere are given by spherical harmonics with $k=l(l+4), l=1,2, \ldots$ and hence $z=l+2$. The $l=1$ harmonic appears to violate the bound (2.8). However, it is straightforward to see that the construction for $l=1$ leads to $d C=0$ for which $C$ can be removed by a transformation of the form (2.5). Thus for $S^{5}$ we should consider $l \geq 2$ leading to solutions with $z=4,5, \ldots$, as above. It is worth pointing out that for higher values of $l$ some of the eigenfunctions will also lead to closed $C$ : if we consider the harmonic function on $\mathbb{R}^{6}$ given by $x^{i_{1}} \ldots x^{i_{l}} c_{i_{1} \ldots i_{l}}$ where $c$ is symmetric and traceless then, with $J=d x^{1} \wedge d x^{2}+d x^{3} \wedge d x^{4}+d x^{5} \wedge d x^{6}$ we see that $d C=0$ if $J_{[i}{ }^{j} c_{k] j i_{3} \ldots i_{l}}=0$.

\footnotetext{
${ }^{2}$ Note that in general the one-form $C$ defined in (2.9) has a component in the $d r$ direction, unlike in (2.6). However, locally we can remove it by a transformation of the form (2.5). Also, one can directly show that the resulting one-form $\beta$ is co-closed on the $S E_{5}$ space.
} 


\subsection{Supersymmetry}

We introduce the frame

$$
\begin{aligned}
e^{+} & =r d x^{+} \\
e^{-} & =r\left(d x^{-}+C\right) \\
e^{2} & =r d x_{1} \\
e^{3} & =r d x_{2} \\
e^{4} & =\frac{d r}{r} \\
e^{m} & =e_{S E}^{m}, \quad m=5, \ldots, 9
\end{aligned}
$$

where $e_{S E}^{m}$ is an orthonormal frame for the $S E_{5}$ space. We can write

$$
\begin{aligned}
& F_{5}=B_{5}+*_{10} B_{5} \\
& B_{5}=4 e^{+} \wedge e^{-} \wedge e^{2} \wedge e^{3} \wedge e^{4}-r e^{+} \wedge d C \wedge e^{2} \wedge e^{3}
\end{aligned}
$$

where we have chosen $\epsilon_{+-23456789}=+1$. The Killing spinor equation can be written

$$
D_{M} \epsilon+\frac{i}{16} \not H \Gamma_{M} \epsilon=D_{M} \epsilon+\frac{i}{2} \not B \Gamma_{M} \epsilon=0 \text {. }
$$

We are using the conventions for type IIB supergravity [42] [43] as in [44] and in particular, $\Gamma_{11}=\Gamma_{+-23456789}$ with the chiral IIB spinors satisfying $\Gamma_{11} \epsilon=-\epsilon$.

If $\epsilon$ are the Killing spinors for the $A d S_{5} \times S E_{5}$ solution, then we find that we must also impose that

$$
\begin{aligned}
\Gamma^{+-23} \epsilon & =i \epsilon \\
\Gamma^{+} \epsilon & =0 .
\end{aligned}
$$

The first condition maintains the Poincaré supersymmetries but breaks all of the superconformal supersymmetries (this can be explicitly checked using, for example, the results of [45]). The second condition breaks a further half of these 3 . Thus when $d C \neq 0$, we preserve two Poincaré supersymmetries for a generic $S E_{5}$ and this is increased to eight Poincaré supersymmetries for $S^{5}$.

\footnotetext{
${ }^{3}$ That we preserve the Poincaré supersymmetries suggests that we can extend our solutions away from the near horizon limit of the D3-branes. This is indeed the case but we won't expand upon that here.
} 


\section{The $D=11$ solutions}

The construction of the $D=11$ solutions is very similar. We consider the ansatz for $\mathrm{D}=11$ supergravity solutions:

$$
\begin{aligned}
d s^{2} & =\frac{d \rho^{2}}{4 \rho^{2}}+\rho^{2}\left[2 d x^{+} d x^{-}+d x^{2}\right]+d s^{2}\left(S E_{7}\right)+2 \rho^{2} C d x^{+} \\
G & =-3 \rho^{2} d x^{+} \wedge d x^{-} \wedge d \rho \wedge d x+d x^{+} \wedge d x \wedge d\left(\rho^{3} C\right)
\end{aligned}
$$

where $S E_{7}$ is a seven-dimensional Sasaki-Einstein space and $d s^{2}\left(S E_{7}\right)$ is normalised so that the Ricci tensor is equal to six times the metric (this is the normalisation of a unit radius seven-sphere). It is convenient to change coordinates via $\rho=r^{2}$ to bring the solution to the form

$$
\begin{aligned}
d s^{2} & =\frac{d r^{2}}{r^{2}}+r^{4}\left[2 d x^{+} d x^{-}+d x^{2}\right]+d s^{2}\left(S E_{7}\right)+2 r^{4} C d x^{+} \\
G & =-6 r^{5} d x^{+} \wedge d x^{-} \wedge d r \wedge d x+d x^{+} \wedge d x \wedge d\left(r^{6} C\right)
\end{aligned}
$$

In these coordinates the cone metric

$$
d s_{C Y}^{2}=d r^{2}+r^{2} d s^{2}\left(S E_{7}\right)
$$

is a metric on Calabi-Yau four-fold. We will use the same notation for the $C Y$ space as in the previous section.

When the one-form $C$ is zero we have the standard $A d S_{4} \times S E_{7}$ solution of $D=11$ supergravity that, in general, preserves eight supersymmetries. We again find that all the equations of motion are solved if $C$ is a one-form on $C Y_{4}$ and the two-form $d C$ is co-closed

$$
d *_{C Y} d C=0
$$

The solutions are again invariant under the transformation (2.5). We will consider solutions where the one-form $C$ has weight $\lambda$ under the action of $r \partial_{r}$, corresponding to dynamical exponent $z=2+\lambda / 2$. As before, using the results in appendix $\mathrm{A}$ of [33], we consider solutions constructed from a co-closed one-form $\beta$ on the $S E_{7}$ space that is an eigenmode of the Laplacian $\Delta_{S E}$ :

$$
C=r^{\lambda} \beta, \quad \Delta_{S E} \beta=\mu \beta, \quad d^{\dagger} \beta=0 .
$$

One can check that $d C$ is co-closed providing that $\mu=\lambda(\lambda+4)$. For our applications we choose the branch $\lambda=-2+\sqrt{4+\mu}$ leading to solutions with

$$
z=1+\frac{1}{2} \sqrt{4+\mu}
$$


A general result valid for any seven-dimensional Einstein space, normalised as we have, is that for co-closed 1 -forms $\mu \geq 12$ and $\mu=12$ holds iff the 1 -form is dual to a Killing vector (see section 4.3 of [34]). Thus in general our construction leads to solutions with

$$
z \geq 3
$$

and the bound is again saturated for all $S E_{7}$ spaces. Observe that the solutions of [31] with $z=3$ fit into this class. Specifically they are obtained by setting $C=\sigma r^{2} \eta$ (after redefining $x \rightarrow x / 2$ and $x^{-} \rightarrow-x^{-} / 8$ ). On $S^{7}$ the spectrum of $\Delta_{S^{7}}$ is well known and we have $\mu=s(s+6)+5$ for $s=1,2,3 \ldots$ (see for example [34] eq (7.2.5)) leading to $\lambda=1+s$ and hence new classes of solutions with $z=3,3 \frac{1}{2}, 4, \ldots$ These solutions come in families transforming in the $S O 8$ ) irreps $\mathbf{2 8}, \mathbf{1 6 0} \mathbf{v}_{\mathbf{v}}, \mathbf{5 6 7}_{\mathbf{v}}, \ldots$. Results on the spectrum of the Laplacian on some homogeneous $S E_{7}$ spaces can be found in [46, 47, ,48].

As before we can construct $(1,1)$ co-closed two-forms $d C$ using scalar functions $\Phi$ on $C Y_{4}$ We write

$$
C_{i}=J_{i}^{j} \partial_{j} \Phi, \quad \nabla_{C Y}^{2} \Phi=\alpha
$$

and $d C$ is again primitive if and only if $\alpha=0$. The solutions of [31] with $z=3$ arise by taking $\Phi=\sigma r^{2}$ and $\alpha=-8 \sigma$ leading to $C=\sigma r^{2} \eta$. We now focus on solutions with $\alpha=0$, corresponding to harmonic functions on the CY cone. We take

$$
\Phi=r^{\lambda} f
$$

where $f$ is a function on the $S E_{7}$ space satisfying

$$
-\nabla_{S E_{7}}^{2} f=k f
$$

with $k=\lambda(\lambda+6)$. For our applications we choose the branch $\lambda=-3+\sqrt{9+k}$ leading to solutions with $z=\frac{1}{2}+\frac{1}{2} \sqrt{9+k}$. For example, on the seven-sphere the eigenfunctions $f$ are given by spherical harmonics with $k=l(l+6)$ with $l=1,2, \ldots$ and hence $z=2+l / 2$. Excluding the $l=1$ harmonic, as it can be removed by a transformation of the form (2.5), for $S^{7}$ we are left with solutions with $z=3,7 / 2,4, \ldots$, as above. 


\subsection{Supersymmetry}

We introduce a frame

$$
\begin{aligned}
e^{+} & =r^{2} d x^{+} \\
e^{-} & =r^{2}\left(d x^{-}+C\right) \\
e^{2} & =r^{2} d x \\
e^{3} & =\frac{d r}{r} \\
e^{m} & =e_{S E}^{m}, \quad m=4, \ldots, 10 .
\end{aligned}
$$

We thus have

$$
\begin{aligned}
G & =6 e^{+} \wedge e^{-} \wedge e^{2} \wedge e^{3}+r^{2} e^{+} \wedge e^{2} \wedge d C \\
*_{11} G & =-6 \operatorname{Vol}\left(S E_{7}\right)+d x^{+} *_{C Y} d C
\end{aligned}
$$

where we have chosen the orientation $\epsilon_{+-23 \ldots 10}=+1$.

The Killing spinor equation can be written as

$$
\nabla_{M} \epsilon+\frac{1}{288}\left[\Gamma_{M}{ }^{N_{1} N_{2} N_{3} N_{4}}-8 \delta_{M}^{N_{1}} \Gamma^{N_{2} N_{3} N_{4}}\right] G_{N_{1} N_{2} N_{3} N_{4}} \epsilon=0 .
$$

We are using the conventions for $D=11$ supergravity [49] as in [50] and in particular $\Gamma_{+-2345678910}=+1$.

If $\epsilon$ are the Killing spinors arising for the $A d S_{4} \times S E_{7}$ solution, then we find that we must also impose that

$$
\begin{aligned}
\Gamma^{+-2} \epsilon & =-\epsilon \\
\Gamma^{+} \epsilon & =0 .
\end{aligned}
$$

The first condition maintains the Poincaré supersymmetries but breaks all of the superconformal supersymmetries. The second condition breaks a further half of these. Thus when $d C \neq 0$, we preserve two Poincaré supersymmetries for a generic $S E_{7}$ and this is increased to eight Poincaré supersymmetries for $S^{7}$.

\subsection{Skew-Whiffed Solutions}

If $A d S_{4} \times S E_{7}$ is a supersymmetric solution of $D=11$ supergravity, then if we "skewwhiff" by reversing the sign of the flux (or equivalently changing the orientation of $S E_{7}$ ) then apart from the special case when the $S E_{7}$ space is the round $S^{7}$, all supersymmetry is broken [51]. Despite the lack of supersymmetry, such solutions are known to be perturbatively stable [51]. Similarly, if we reverse the sign of the flux in our new solutions (3.2), we will obtain solutions of $D=11$ supergravity that will generically not preserve any supersymmetry. 


\section{Further Generalisation}

We now discuss a further generalisation of the solutions that we have considered so far, preserving the same amount of supersymmetry, which incorporate the construction of [24]. For type IIB the metric is now given by

$$
d s^{2}=\frac{d r^{2}}{r^{2}}+r^{2}\left[2 d x^{+} d x^{-}+d x_{1}^{2}+d x_{2}^{2}\right]+d s^{2}\left(S E_{5}\right)+r^{2}\left[2 C d x^{+}+h\left(d x^{+}\right)^{2}\right]
$$

with the five-form unchanged from (2.1). The conditions on the one-form $C$ are as before and we demand that $h$ is a harmonic function on the $C Y_{3}$ cone:

$$
\nabla_{C Y}^{2} h=0
$$

Choosing $h$ to have weight $\lambda^{\prime}$ under $r \partial_{r}$ we take

$$
h=r^{\lambda^{\prime}} f^{\prime}
$$

where $f^{\prime}$ is an eigenfunction of the Laplacian on $S E_{5}$ with eigenvalue $k^{\prime}$

$$
-\nabla_{S E_{5}}^{2} f^{\prime}=k^{\prime} f^{\prime}
$$

with $k^{\prime}=\lambda^{\prime}\left(\lambda^{\prime}+4\right)$. If we set $C=0$ and choose the branch $\lambda^{\prime}=-2+\sqrt{4+k^{\prime}}$ then these are the solutions constructed in section 5 of [24] and have dynamical exponent

$z=\frac{1}{2} \sqrt{4+k^{\prime}}$. As noted in [24] an application of Lichnerowicz's theorem [52], [53] implies that these solutions have $z \geq 3 / 2$ with $z=3 / 2$ only possible for $S^{5}$. Now if there is a scalar eigenfunction with eigenvalue $k^{\prime}$ and a one-form eigenmode of the Laplacian on $S E_{5}$ with eigenvalue $\mu$ that satisfy $z=\frac{1}{2} \sqrt{4+k^{\prime}}=1+\sqrt{1+\mu}$ then we can superpose the solution with $h$ as in (4.3) and the one-form $C$ as in (2.6) and have a solution with scaling symmetry with this value of $z$. For example on $S^{5}$, using the notation as before, we have $k^{\prime}=l^{\prime}\left(l^{\prime}+4\right), l^{\prime}=1,2, \ldots$ and $\mu=(s+1)(s+3)$, $s=1,2, \ldots$ and hence we must demand that $l^{\prime}=2(s+2), s=1,2, \ldots$, giving solutions with $z=3+s$.

The story for $D=11$ is very similar. The metric is now given by

$$
d s^{2}=\frac{d r^{2}}{r^{2}}+r^{4}\left[2 d x^{+} d x^{-}+d x^{2}\right]+d s^{2}\left(S E_{7}\right)+r^{4}\left[2 C d x^{+}+h\left(d x^{+}\right)^{2}\right]
$$

with the four-form unchanged from (3.2). The conditions on the one-form $C$ are as before and we demand that $h$ is a harmonic function on the $C Y_{4}$ cone:

$$
\nabla_{C Y}^{2} h=0
$$


Choosing $h$ to have weight $\lambda^{\prime}$ under $r \partial_{r}$ we take

$$
h=r^{\lambda^{\prime}} f^{\prime}
$$

where $f^{\prime}$ is an eigenfunction of the Laplacian on $S E_{7}$ with eigenvalue $k^{\prime}$

$$
-\nabla_{S E_{7}}^{2} f^{\prime}=k^{\prime} f^{\prime}
$$

with $k^{\prime}=\lambda^{\prime}\left(\lambda^{\prime}+6\right)$. If we set $C=0$ and chose the branch $\lambda^{\prime}=-3+\sqrt{9+k^{\prime}}$ then these solutions have dynamical exponent $z=\frac{1}{4}\left(1+\sqrt{9+k^{\prime}}\right)$. Lichnerowicz's theorem [52],53] implies that these solutions have $z \geq 5 / 4$ with $z=5 / 4$ only possible for $S^{7}$. If there is a scalar eigenfunction with eigenvalue $k^{\prime}$ and a one-form eignemode of the Laplacian on $S E_{7}$ with eigenvalue $\mu$ that satisfy $z=\frac{1}{4}\left(1+\sqrt{9+k^{\prime}}\right)=1+\frac{1}{2} \sqrt{4+\mu}$ then we can superpose the solution with $h$ as in (4.7) and the one-form $C$ as in (3.5) and have a solution with scaling symmetry with this value of $z$. For example on $S^{7}$, using the notation as before, we have $k^{\prime}=l^{\prime}\left(l^{\prime}+6\right), l^{\prime}=1,2, \ldots$ and $\mu=s(s+6)+5$, $s=1,2, \ldots$ and hence we must demand that $l^{\prime}=2(s+3), s=1,2, \ldots$, giving solutions with $z=\frac{1}{2}(5+s)$.

\section{Acknowledgements}

We would like to thank Seok Kim, James Sparks, Oscar Varela and Daniel Waldram. for helpful discussions. JPG is supported by an EPSRC Senior Fellowship and a Royal Society Wolfson Award.

\section{References}

[1] D. T. Son, "Toward an AdS/cold atoms correspondence: a geometric realization of the Schroedinger symmetry," Phys. Rev. D 78 (2008) 046003 arXiv:0804.3972 [hep-th]].

[2] K. Balasubramanian and J. McGreevy, "Gravity duals for non-relativistic CFTs," Phys. Rev. Lett. 101, 061601 (2008) arXiv:0804.4053 [hep-th]].

[3] M. Sakaguchi and K. Yoshida, "Super Schrodinger in Super Conformal," arXiv:0805.2661 [hep-th].

[4] W. D. Goldberger, "AdS/CFT duality for non-relativistic field theory," arXiv:0806.2867 [hep-th]. 
[5] J. L. B. Barbon and C. A. Fuertes, "On the spectrum of nonrelativistic AdS/CFT," JHEP 0809, 030 (2008) [arXiv:0806.3244 [hep-th]].

[6] M. Sakaguchi and K. Yoshida, "More super Schrodinger algebras from psu(2,2-4)," JHEP 0808, 049 (2008) [arXiv:0806.3612 [hep-th]].

[7] W. Y. Wen, "AdS/NRCFT for the (super) Calogero model," arXiv:0807.0633 [hep-th].

[8] C. P. Herzog, M. Rangamani and S. F. Ross, "Heating up Galilean holography," JHEP 0811, 080 (2008) [arXiv:0807.1099 [hep-th]].

[9] J. Maldacena, D. Martelli and Y. Tachikawa, "Comments on string theory backgrounds with non-relativistic conformal symmetry," JHEP 0810, 072 (2008) arXiv:0807.1100 [hep-th]].

[10] A. Adams, K. Balasubramanian and J. McGreevy, "Hot Spacetimes for Cold Atoms," JHEP 0811, 059 (2008) arXiv:0807.1111 [hep-th]].

[11] Y. Nakayama, "Index for Non-relativistic Superconformal Field Theories," JHEP 0810, 083 (2008) [arXiv:0807.3344 [hep-th]].

[12] D. Minic and M. Pleimling, "Non-relativistic AdS/CFT and Aging/Gravity Duality," arXiv:0807.3665 [cond-mat.stat-mech].

[13] J. W. Chen and W. Y. Wen, "Shear Viscosity of a Non-Relativistic Conformal Gas in Two Dimensions," arXiv:0808.0399 [hep-th].

[14] A. V. Galajinsky, "Remark on quantum mechanics with conformal Galilean symmetry," Phys. Rev. D 78, 087701 (2008) arXiv:0808.1553 [hep-th]].

[15] S. Kachru, X. Liu and M. Mulligan, "Gravity Duals of Lifshitz-like Fixed Points," Phys. Rev. D 78, 106005 (2008) [arXiv:0808.1725 [hep-th]].

[16] S. S. Pal, "Null Melvin Twist to Sakai-Sugimoto model," arXiv:0808.3042 [hepth].

[17] S. Sekhar Pal, "Towards Gravity solutions of AdS/CMT," arXiv:0808.3232 [hepth].

[18] S. Pal, "More gravity solutions of AdS/CMT," arXiv:0809.1756 [hep-th]. 
[19] P. Kovtun and D. Nickel, "Black holes and non-relativistic quantum systems," arXiv:0809.2020 [hep-th].

[20] C. Duval, M. Hassaine and P. A. Horvathy, "The geometry of Schródinger symmetry in gravity background/non-relativistic CFT," arXiv:0809.3128 [hep-th].

[21] S. S. Lee, "A Non-Fermi Liquid from a Charged Black Hole: A Critical Fermi Ball," arXiv:0809.3402 [hep-th].

[22] D. Yamada, "Thermodynamics of Black Holes in Schroedinger Space," arXiv:0809.4928 [hep-th].

[23] F. L. Lin and S. Y. Wu, "Non-relativistic Holography and Singular Black Hole," arXiv:0810.0227 [hep-th].

[24] S. A. Hartnoll and K. Yoshida, "Families of IIB duals for nonrelativistic CFTs," arXiv:0810.0298 [hep-th].

[25] M. Schvellinger, "Kerr-AdS black holes and non-relativistic conformal QM theories in diverse dimensions," JHEP 0812, 004 (2008) [arXiv:0810.3011 [hep-th]].

[26] L. Mazzucato, Y. Oz and S. Theisen, "Non-relativistic Branes," arXiv:0810.3673 [hep-th].

[27] M. Rangamani, S. F. Ross, D. T. Son and E. G. Thompson, "Conformal nonrelativistic hydrodynamics from gravity," arXiv:0811.2049 [hep-th].

[28] A. Akhavan, M. Alishahiha, A. Davody and A. Vahedi, "Non-relativistic CFT and Semi-classical Strings," arXiv:0811.3067 [hep-th].

[29] A. Adams, A. Maloney, A. Sinha and S. E. Vazquez, "1/N Effects in NonRelativistic Gauge-Gravity Duality," arXiv:0812.0166 [hep-th].

[30] M. Taylor, "Non-relativistic holography," arXiv:0812.0530 [hep-th].

[31] J. P. Gauntlett, S. Kim, O. Varela and D. Waldram, "Consistent supersymmetric Kaluza-Klein truncations with massive modes," arXiv:0901.0676 [hep-th].

[32] S. Pal, "Anisotropic gravity solutions in AdS/CMT," arXiv:0901.0599 [hep-th].

[33] D. Martelli and J. Sparks, "Symmetry-breaking vacua and baryon condensates in AdS/CFT," arXiv:0804.3999 [hep-th]. 
[34] M. J. Duff, B. E. W. Nilsson and C. N. Pope, "Kaluza-Klein Supergravity," Phys. Rept. 130 (1986) 1.

[35] H. J. Kim, L. J. Romans and P. van Nieuwenhuizen, "The Mass Spectrum Of Chiral N=2 D=10 Supergravity On S**5," Phys. Rev. D 32 (1985) 389.

[36] A. Ceresole, G. Dall'Agata and R. D'Auria, "KK spectroscopy of type IIB supergravity on AdS(5) x T(11)," JHEP 9911 (1999) 009 [arXiv:hep-th/9907216].

[37] J. P. Gauntlett, D. Martelli, J. Sparks and S. T. Yau, "Obstructions to the existence of Sasaki-Einstein metrics," Commun. Math. Phys. 273 (2007) 803 arXiv:hep-th/0607080.

[38] S. S. Gubser, "Einstein manifolds and conformal field theories," Phys. Rev. D 59 (1999) 025006 arXiv:hep-th/9807164.

[39] G. W. Gibbons, S. A. Hartnoll and Y. Yasui, "Properties of some five dimensional Einstein metrics," Class. Quant. Grav. 21 (2004) 4697 arXiv:hep-th/0407030.

[40] H. Kihara, M. Sakaguchi and Y. Yasui, "Scalar Laplacian on Sasaki-Einstein manifolds Y(p,q)," Phys. Lett. B 621 (2005) 288 arXiv:hep-th/0505259.

[41] J. P. Gauntlett, D. Martelli, J. Sparks and D. Waldram, "Sasaki-Einstein metrics on S(2) x S(3)," Adv. Theor. Math. Phys. 8 (2004) 711 [arXiv:hep-th/0403002].

[42] J. H. Schwarz, "Covariant Field Equations Of Chiral N=2 D=10 Supergravity," Nucl. Phys. B 226 (1983) 269.

[43] P. S. Howe and P. C. West, "The Complete N=2, D=10 Supergravity," Nucl. Phys. B 238 (1984) 181.

[44] J. P. Gauntlett, D. Martelli, J. Sparks and D. Waldram, "Supersymmetric AdS(5) solutions of type IIB supergravity," Class. Quant. Grav. 23 (2006) 4693 arXiv:hep-th/0510125.

[45] H. Lu, C. N. Pope and J. Rahmfeld, "A construction of Killing spinors on S**n," J. Math. Phys. 40, 4518 (1999) arXiv:hep-th/9805151].

[46] D. Fabbri, P. Fre, L. Gualtieri and P. Termonia, "M-theory on AdS(4) x M(111): The complete $\operatorname{Osp}(2-4)$ x SU(3) x SU(2) spectrum from harmonic analysis," Nucl. Phys. B 560 (1999) 617 arXiv:hep-th/9903036. 
[47] P. Merlatti, "M-theory on AdS(4) x Q(111): The complete Osp(2-4) x SU(2) x SU(2) x SU(2) spectrum from harmonic analysis," Class. Quant. Grav. 18 (2001) 2797 arXiv:hep-th/0012159.

[48] P. Termonia, "The complete $\mathrm{N}=3$ Kaluza-Klein spectrum of 11D supergravity on AdS(4) x N(010)," Nucl. Phys. B 577 (2000) 341 arXiv:hep-th/9909137.

[49] E. Cremmer, B. Julia and J. Scherk, "Supergravity theory in 11 dimensions," Phys. Lett. B 76 (1978) 409.

[50] J. P. Gauntlett and S. Pakis, "The geometry of D = 11 Killing spinors," JHEP 0304 (2003) 039 arXiv:hep-th/0212008.

[51] M. J. Duff, B. E. W. Nilsson and C. N. Pope, "The Criterion For Vacuum Stability In Kaluza-Klein Supergravity," Phys. Lett. B 139 (1984) 154.

[52] A. Lichnerowicz, "Géometrie des groupes de transformations," Dunod, Paris, 1958.

[53] M. Obata, "Certain conditions for a Riemannian manifold to be isometric to a sphere," J. Math. Soc. Japan 14 (1962) 333-340. 